\title{
ENCADENAMIENTO PRODUCTIVO, ESTADO ACTUAL DEL CLÚSTER AUTOMOTRIZ EN LA REGIÓN DE SAN LUIS POTOSÍ ${ }^{1}$
}

\author{
Lucila Patricia Cruz Covarrubias ${ }^{2}$ \\ Pedro Aguilar Pérez ${ }^{3}$
}

Para citar este artículo: Cruz, L., y Aguilar, P. (2015). Encadenamiento productivo, estado actual del clúster automotriz en la región de San Luis Potosí. Inquietud Empresarial. Vol. XV (2), 37-64.

Fecha de recepción: 30 de junio de 2015 Fecha de aceptación: 4 de diciembre de 2015

\footnotetext{
Artículo de investigación científica y tecnológica como resultado del proyecto de investigación "articulación productiva del sector automotriz en la región Centro Occidente de México, implicaciones económicas y sociales para impulsar el crecimiento".

2 Profesora de tiempo completo de la Universidad de Guadalajara, Jalisco, México con Maestría en Auditoría. Coordinador del Cuerpo Académico "Liderazgo y habilidades directivas en la gestión empresarial" UDG-CA669, patriciacruz2204@hotmail.com

3 Profesor e Investigador de la Universidad de Guadalajara, adscrito al Departamento de Administración del Centro Universitario de Ciencias Económico Administrativas. Maestría en Mercadotecnia. Artículo del proyecto de investigación "Articulación productiva del sector automotriz en la región Centro Occidente de México, implicaciones económicas y sociales para impulsar el crecimiento" que se realizó en 2003-2004, paguilar_17@hotmail.com
} 


\title{
Resumen
}

En la actualidad, el encadenamiento productivo es una condición indispensable para el desarrollo económico local en el nuevo escenario global y competitivo. En San Luis Potosí empresas e instituciones públicas y privadas han desarrollado acciones de cooperación e interacción para la formación del clúster automotriz. El trabajo analiza y describe la acción de la cadena productiva, con el objetivo de examinar las características de la acción conjunta de estos sectores y el grado de consolidación del clúster. La investigación aborda un método cualitativo, se sustenta en la técnica de entrevista semiestructurada. Conclusiones importantes encontradas son: en la región potosina encontramos un clúster automotriz en formación. Tanto la cadena productiva como las instituciones de apoyo al clúster requieren ser fortalecidas.

Palabras claves: cadena productiva, clúster, industria automotriz, vinculación.

\section{Productive chain, current status of the automotive cluster in the region of San Luis Potosi}

\begin{abstract}
Currently, the production chain is an indispensable condition for local economic development, regarding a global competitive scenario. At San Luis Potosi, public and private institutions have developed cooperative and interactional actions in order to formalize an automotive cluster. This research paper analyzes and describes the action of the productive chain, in contemplation of examining the characteristics of the joint action of these sectors and the degree of consolidation within the cluster. The research is following the principles of a qualitative method, based on the semistructured interview technique. Important conclusions assess: in the Potosi region an automotive cluster development was found. Both, the production chain and support institutions, are vital for the strengthening of the corporations in this area.
\end{abstract}

Key words: supply chain, cluster, auto industry, entailment. 


\section{Encadeamneto produtivo, estado atual do clúster automotriz na região de San Luis Potosi}

\section{Resumo}

$\mathrm{Na}$ atualidade, o encadeamneto produtivo é uma condição indispensável para o desenvolvimento econômico local no novo cenário global e competitivo. Em San Luis Potosí empresas e instituições públicas e privadas tem desenvolvido ações de cooperação e interação para a formação do clúster automotriz. O trabalho analisa e descreve a ação da cadeia produtiva, com o objetivo de examinar as características da ação conjunta destes setores e o grau de consolidação do clúster. A investigação aborda um método qualitativo, se sustenta na técnica de entrevista semi-estruturada. Conclusões importantes encontradas são: na região potosina encontramos um clúster automotriz em formação. Tanto a cadeia produtiva como as instituições de apoio ao clúster requerem ser fortalecidas.

Palavras chave: cadeia produtiva, clúster, industria automotriz, vinculação. 


\section{Introducción}

En este nuevo siglo se ha instalado en el sector empresarial y en las autoridades gubernamentales una nueva forma de analizar la actividad industrial y los encadenamientos que se desarrollan entre las distintas fases de los procesos productivos, a partir de los cuales se podrían definir las nuevas políticas económicas. Esta actividad industrial está denominada por lo que se conoce como clúster.

El proceso de globalización, la demanda de vehículos en el orbe, la regulación gubernamental y el cambio tecnológico han modificado las estrategias de relación, vinculación y de operación desarrolladas por las empresas automotrices. Ante esto, la industria automotriz de México y específicamente del Estado de San Luis Potosí (SLP), representa un sector clave en su estrategia de modernización e integración a la economía mundial. Este sector productivo es uno de los más dinámicos debido a las remuneraciones que genera, su participación en el comercio exterior, ampliación de su formación de capital, valor que agrega a sus productos e integración con otros sectores industriales y de servicios.

En este sentido, para toda economía es importante la identificación y evaluación del desempeño de sus cadenas productivas locales, ya que esto permite integrar o consolidar los eslabones productivos de una región (Dávila, 2002). Además, el análisis de la constitución de cadenas productivas (clúster automotriz regional) permite establecer el tipo de ventajas competitivas que tiene una localidad, así como los flujos de transmisión del conocimiento y su potencial para la innovación.

Otra justificación del estudio es que constituye una oportunidad para analizar las dimensiones de un sector industrial: por un lado, las acciones que realizan las empresas y los encadenamientos productivos locales que se generan y, por otro, las iniciativas que desarrolla el gobierno para fomentar la inversión y provocar desarrollos locales, así como la vinculación con instituciones educativas para la formación de capital humano. A nivel nacional son escasos los trabajos que combinan estos ámbitos.

Partiendo de este contexto y ubicándonos en el terreno de la investigación local las preguntas de investigación que se intentan responder son: ¿Cuáles son las características actuales del clúster automotrices en SLP? y ¿Cuál es la situación de vinculación que existe entre las empresas automotrices, instituciones gubernamentales y de educación?

Por ello, en este trabajo, nos proponemos analizar las particularidades del clúster del sector automotriz en la región de SLP, a partir de los resultados de una investigación 
que permite exponer conclusiones significativas. El objetivo principal es: conocer y analizar las interacciones que existen entre los actores de la cadena productiva en el sector automotriz de la región potosina, así como, exponer aquellos factores que nos permiten evaluar si el clúster, tal como se denomina formalmente, ha experimentado o no un fortalecimiento.

Los objetivos señalados se vinculan con exposiciones teóricas vigentes en la discusión internacional sobre el papel de los agrupamientos económicos o clúster, y con la necesidad de incluir en las políticas públicas actores diversos que actúan de manera conjunta en empresas, cadenas productivas, redes e instituciones que se conectan de distintas maneras (Yoguel, (2000); Stumpo, (2005); Casas, (2004); citados por Hualde y Gomis, (2007).

El método de investigación se aborda desde un enfoque cualitativo. Los datos presentados se basan en entrevistas realizadas en el periodo de noviembre de 2013 a junio de 2014 a directivos, empresarios y/o representantes de 10 empresas automotrices: armadora establecida en el Estado, General Motors [GM], 9 proveedores, Así mismo, a directivos de la Secretaría de Desarrollo Económico del Estado de San Luis Potosí [SEDECO], y de dos instituciones de educación pública: Universidad Politécnica de San Luis Potosí y CONALEP-SLP.

\section{Concepto de Clúster}

El término clúster, de acuerdo con varios autores, es muy variable, ya sea por su amplitud y/o elasticidad que hace que pueda ser aplicado a cualquier sector productivo que establezcan entre sí un determinado vínculo.

Por ello, desde la década de los ochenta se establecen distintos enfoques económicos que se han ocupado del estudio de este tipo de aglomeración económica. Por señalar algunas de las corrientes y con base a (Dahl, 2001 citado en Gil, 2011, p. 283), son:

- La economía de la innovación, en particular, el análisis de los sistemas nacionales, regionales y sectores de la innovación. Dentro de este enfoque se encuentran autores como Nelson (1993); Freeman (1997) y Bresch y Malerba (2006).

- Geografía económica, principalmente la variante neomarshaliana. Se destacan autores como Scott (2000), Stroper (1997) y Martín y Sunley (2003).

- Las nuevas teorías del conocimiento de crecimiento económico instauradas por autores como Grossman y Helpman (1991).

- La nueva geografía económica, preconizada por Krugman (2000), Venables (1996) y Fujita (1999). 
- La de ventaja competitiva y la teoría de cúmulos y competencia, elaborada por Porter (1991, 2003).

Ante estos modelos teóricos, las actividades de las empresas de un sector determinado afectan el desempeño de otros sectores de la región a través de sus demandas de insumos, de la difusión de nuevas tecnologías y de su relación comercial ya que comparten un mercado común (Feser y Bergman, 2000). En este sentido, Porter (2003) afirma que el mapa económico mundial está dominado por lo que se denominan clústers o agrupamientos económicos, los cuales denomina como "concentraciones geográficas de empresas interconectadas, suministradores especializados, proveedores de servicios, empresas de sectores afine e instituciones conexas (por ejemplo, universidades, institutos de normalización, asociaciones comerciales) que compiten pero también cooperan" (Gil, 2011, p. 284). Además, Porter alude que esta agrupación de empresas e instituciones, es una actividad característica de la mayoría de las economías nacionales y regionales, en especial las de los países más adelantados.

Hualde y Gomis (citando a Boscherini y Poma, 2000). Señalan que los “clúster, al igual que los distritos industriales (Beccattini, 1994), redes (Salman y Saives, 2005) o los sistemas regionales de innovación (Cooke, 2004) se caracterizan por una serie de interacciones entre diferentes actores: como las propias empresas que establecen dinámicas conjuntas de cooperación y competencia; entre instituciones educativas o centros de investigación; y entre las asociaciones y los gobiernos locales para la creación de redes o asociaciones de carácter formal o informal" (2007, p. 196).

Por otro lado, (Otero at al., 2004) mencionado en y Vera Garnica Ganga Contreras (2007), refieren que los clúster son "redes de producción de empresas fuertemente interdependientes (incluyendo proveedores especializados), ligadas unas a otras en una cadena de producción que añade valor" (p. 306), así, los autores acuerdan que el concepto de clúster va más allá de las redes horizontales simples, en las cuales las organizaciones que operan en el mismo mercado de productos finales y pertenecen al mismo sector productivo cooperan en ciertas áreas, estableciendo alianzas estratégicas con instituciones de educación, de investigación, de servicios intensivos en conocimiento, instituciones puentes (comisionistas, consultores y clientes).

Al respecto, la consolidación de clúster, concebido como una estrategia para generar valor y competitividad, involucra a empresas encadenadas a una misma actividad además potencializa diferentes áreas de negocio e involucra instituciones de educación e investigación. 
El principal objetivo de un encadenamiento empresarial en el clúster se ha convertido en propiciar el aumento de la competitividad de las organizaciones, consolidar su presencia tanto en el mercado nacional como en el internacional e incrementar su participación en las importaciones (Secretaría de Hacienda y Crédito Público, NAFIN, SHCP, 2011).

Según Örjan Sölvell (2009) mencionado en Flores Perea (2013, p. 34), ilustra que las características centrales de un clúster son:

- Concentración geográfica de la actividad económica; que haya proximidad física entre los actores.

- Especialización en un sector económico concreto; que existe una actividad eje bajo la cual confluyan los actores participantes.

- Efecto derrame, que la actividad beneficie a toda la región, y que involucre a otras actividades con potencial para generar desarrollo regional.

- Tripe Hélice: sistema administración-universidad-empresa.

- Equilibrio entre competencia y colaboración por parte de sus miembros, que haya equidad en trabajo y en la toma de decisiones.

Flores Perea (2013) señala que la conformación del clúster implica directamente;

"el establecimiento de canales de comunicación en función de las necesidades específicas del sector económico del cual se trate, implica ubicar las necesidades de cada uno de los actores a distintos niveles, también implica generar condiciones para trabajar bajo economías de escala y la búsqueda de mejoramiento continuo en procesos y comercialización, así como la puesta en marcha de planes y estrategias a mediano y largo plazo, por lo que se dice los clúster son puertas de acceso a recursos económicos, recursos tecnológicos y mercados" ( p. 35).

A su vez, los clúster generan vínculos de empatía que permitan entender la situación de cada uno de los entes a fin de generar estrategias para potencializar el avance del mismo. Los clúster representan una nueva forma de enfrentar la competencia internacional, al constituir un medio para que las organizaciones generen canales de crecimiento y productividad a través de su integración productiva y social dentro de un espacio geográfico en el que operan. 
La constitución de un clúster implica conocimiento amplio de la producción (bienes y servicios) del sector económico, del comercio y de la vinculación directa e indirecta con todos los entes del clúster. Este proceso inicia por establecer relaciones informales de acercamiento, alrededor de las cuales colaborando juntos se llega después a formalizar y fortalecer diferentes relaciones de cooperación, tales como cooperación horizontal, vertical e institucional.

La cooperación horizontal se refiere a empresas del mismo sector que pueden lograr economías de escala, y por lo tanto mayor poder de negociación; mayor poder de obtener asistencia técnica y organizativa de los productos que interesa aumentar su competitividad, procurando asegurar beneficios proporcionales para todas las empresas (Lara, 2010).

La cooperación vertical, involucra a empresas de sectores afines por dimensión y formas operativas, que consiste en establecerse en el mercado, con el resultado de un trabajo común entre empresas. Ventas en común es la parte más frecuente de este tipo de cooperación vertical. Además también se recurre a la consultoría, a la asistencia de crédito, asesoría por empresas, por lo que las asociaciones empresariales juegan y ejercen un papel determinante en el clúster (Lara, 2010).

Es necesario resaltar que la cooperación institucional es fundamental en la estructura del clúster, las instituciones educativas proporcionan mecanismos de investigación e innovación, las instituciones de gobierno son mediadoras para facultar apoyos de carácter legal, económico y de capacitación, las asociaciones sociales y civiles privadas ponen a disposición toda una estructura organizacional para apoyos y beneficios económicos, tecnológicos y políticos.

Según el modelo de M. Porter en la década de los noventa, sostiene que para que una actividad sea competitiva es necesaria la interconexión de actividades para generar sinergias que permitan la asociatividad a fin de generar economías de escala (Flores Perea, 2013).

Dentro de la dinámica del clúster se incluye un intenso intercambio de información de los negocios, Know-how (conocimiento) y experiencias tecnológicas (Sölvell, 2009). En la integración de un clúster sea del sector que se trate se da de manera nata la participación de distintos actores a diversos niveles, la empresa, asociaciones industriales, instituciones educativas y de investigación, organizaciones de la sociedad civil, gobierno, por mencionar algunos.

La conformación de un clúster regional obedece a la necesidad de un incremento en la competitividad ya que este tipo de trama productivo se instituye como generadoras 
de empleo y riqueza, además de que fomentarán la capacidad de innovación y desarrollo.

\section{Clúster automotriz en San Luis Potosí}

En el caso mexicano, se ha experimentado desarrollos constantes de clúster automotriz en diferentes Estados de la República, como de regiones, algunos han tenido crecimientos e integración exitosas, por citar algunos ejemplo, el clúster automotriz de Nuevo León y recientemente el del Estado de Guanajuato. Autores como (Ungen et al., 2003) citado en Moreno A, et al. (2008) mencionan que en México el desarrollo del sector automotriz en las últimas tres décadas ha sido cuestionado su competitividad, este debate ha ido a la par con un crecimiento exportador y de inversión extranjera directa que no disminuye. Siguiendo con los autores, Ungen et al., aluden que hay una realidad en el sector automotriz de desarrollo y crecimiento muy notable, donde hay "...crecientes flujos de IED, exitoso despegue exportador y nada despreciable generación de empleos" (Moreno Mata et al., 2008, p. 20).

Siguiendo con Ungen et al. (2003) este autor señala (interpreta Moreno A, et al., 2008), que:

"Es posible que desde fuera se vea con más claridad que el país es una localización ideal por reunir atributos competitivos para el sector automotriz, lo mismo que para otros sectores: abundancia de recursos naturales y energéticos (dejando aparte la escasez de gas y otras carencias puntuales de infraestructura); mano de obra barata, dócil, fácil para ser entrenada; cercanía con EEUU, y otros." (p. 20).

A pesar de lo anterior, la integración de clústers regionales en la industria del automóvil ha sido una tendencia común en los países en que las grandes transnacionales automotrices se instalan. El desarrollo de encadenamientos locales o regionales es en cierta forma natural y los casos de más éxito dependen de aprovechar también las capacidades industriales ya existentes con antelación, asociados a la nueva actividad (Katz 1999; Constantino y Lara 2000 en Moreno A, 2008).

Ante este panorama, el Estado de San Luis Potosí no ha quedado ajeno, La industria automotriz es un sector clave de la estrategia de modernización e integración del Estado en la economía mundial. Actualmente este sector, es una de las principales ramas del sector manufacturero de SLP, por las retribuciones que genera, su participación en el comercio exterior, ampliación de su formación de capital y valor agregado a sus bienes y servicios. El desarrollo sostenido de esta industria, ha 
permitido que se integre un clúster automotriz que consolida y proyecta a San Luis Potosí como uno de los centro de proveeduría y producción más importantes del país con proyección internacional.

Esto no hubiera sido posible en el desarrollo de la fortalezas con que las cuenta San Luis Potosí, relacionadas con un gobierno facilitador, geográfica con gran conectividad: fácil acceso a mercados y materias primas; amplia oferta de mano de obra calificada, ambiente laboral estable; industria de autopartes diversificada y un programa estímulos fiscales, que brindan estabilidad económica y social a los empresarios que deciden instalarse en nuestro territorio.

El grado de especialización en la rama de autopartes en San Luis Potosí, así como la dinámica del sector en cuanto a incremento en la inversión privada, hace que se constituya como un clúster con amplias ventajas a nivel regional y nacional inicialmente en autopartes y actualmente en el de la industria terminal de automóviles.

Es preciso señalar que la participación y la cooperación conjunta entre las grandes empresas que conforman un sector industrial en el Estado, estimula una reacción encadenada a favor del sistema productivo del Estado de San Luis Potosí, al favorecer el nacimiento de nuevas empresas, asegurar la especialización productiva, e incrementar la participación comercial a nivel internacional.

\section{Características de la industria automotriz en San Luis Potosí}

La industria automotriz y la de autoparte del Estado de SLP, es fundamental en la estrategia de modernización o integración a la economía mundial y es un sector que ha impulsado fuertemente las últimas dos administraciones del Estado.

La Secretaría de Economía del Estado de SLP [SEDECO], señala que en la primera década del siglo XXI, los altos estándares de calidad convirtieron a SLP en una plataforma de promoción y comercialización de la mayor importancia para cualquier empresa hacia los diversos mercados. Durante este periodo, 50 empresas proveedoras y una armadora automotriz (SEDECO, 2014).

Más recientemente, durante la presente administración estatal, la actividad automotriz registró un crecimiento sin precedentes. Se instalaron 50 nuevas empresas, y se concretaron 20 ampliaciones en el sector, con una inversión total de 39.4 mil millones de pesos y la generación de 21 mil empleos permanentes. Cifras a las que se suma el 
reciente anuncio de BMW Group de instalar en San Luis Potosí su primera planta armadora en México con una inversión de más de mil millones de dólares y la generación de mil quinientos empleos. (SEDECO, 2014a).

En el 2014, el universo de la industria automotriz es de 151 empresas, y 131 proveedores, otorga la novena posición nacional en la fabricación de equipo de transporte y representa con el 57 por ciento de la actividad de exportación total del Estado; participa con alrededor de la $5^{\circ}$ parte de la producción Bruta Total Manufacturera Estatal y ocupa el $11^{\circ}$ lugar por su participación a la Producción Bruta Total Nacional de la Industria Automotriz (SEDECO, 2014b).

Tiene operación directa en los países de Alemania, Arabia Saudita, Brasil, Canadá, China, Corea, Dinamarca, Estados Unidos de América, Francia, Holanda, Inglaterra, Japón y Turquía.

Un gran número de empresas están vinculadas a importantes corporativos nacionales o extranjeros. Algunas de ellas son: "Continental Tire de México; Contitech Mexicana; Cummins; Eaton Truck Componentes; Eptec; Macnp Mexicana; Remy Componentes; Remy Remanufacturing de México; Robert Bochs México Sistemas Automotrices; San Luis Metal Forming; Thyssen Krupp Bilstein Sasa; Toyota Gosei Automotive Sealing México; Valeo Sistemas Eléctricos (División Alternadores); Valeo Sistemas Eléctricos (División Térmico Motor); Valeo Sistemas Eléctricos (División Transmisiones), entre otros más” (SEDECO, 2012).

Entre sus productos destacan vehículos ligeros, motores, rines automotrices, cámaras llantas, cables electrónicos y de telecomunicaciones, a meses, alternativas, embragues, transmisiones, barra estabilizadores, resortes, ensambles de mangueras de hule para sistemas de calefacción automotriz, tubería para sistemas de dirección automotriz, bolsas grandes de aire (bogsprings), mangueras para radiador, manguillas, suspensión para asiento, tubería de acero al carbón, bielas, dámpares, filtros, poleas, amortiguadores de aire, bandas de transmisión y transportadoras, escapes, silenciadores, frenos para auto, sensor de velocidad, mangueras de hule reforzada y sin reforzar, tubos de plásticos. Tubos de plásticos con accesorios y marchas automotrices.

Gracias al desarrollo de fortalezas con las que cuenta el Estado de SLP, así como a las gestiones realizadas por la administración estatal con la participación de funcionarios de los tres niveles de gobierno y de la iniciativa privada, fue que se concretó el arribo de BMW Group a México y a la región potosina. 
La Secretaría refiere, que en general son cuatro características que destacan en las compañías de SLP: 1) el capital de 48 empresas fue cien por ciento extranjero; 2) existen 38 empresas certificadas bajo la norma ISO/TS $16949^{4}$; 3) el 52.9\% de los insumos que utilizaron las empresas en la generación de sus productos es de origen nacional, y 4) se instaló en el Estado (Municipio de Villa de Reyes) la empresa de ensamble automotriz: General Motors de México, (Complejo San Luis Potosí) ubicado en el municipio de Villa de Reyes, cuyas principales características son las siguientes (tabla 2):

\section{Metodología}

El método de investigación se aborda desde un enfoque cualitativo a partir de una primera fase de revisión documental para analizar el concepto de clúster y de la situación del sector automotriz en el Estado de SLP, a través de artículos en revistas científicas, libros, tesis, informes, y su relación con los implicados en el tema que nos compete. En la segunda fase se realizó investigación de campo en la que se recolectó información empírica a través de la entrevista semiestructurada, que como lo indica la técnica, la parte libre permite profundizar en las características específicas de los entrevistados. Por ello, admite una mayor libertad y flexibilidad en la obtención de información (Hernández, R., et al, 2010). Se contactó en esta instancia de la investigación a diez directivos de empresas del sector automotriz todas establecidas en la ciudad de San Luis Potosí. La investigación se llevó a cabo en los meses de enero a abril de 2014, una institución del Gobierno estatal y dos instituciones educativas públicas.

La entrevista fue aplicada mediante un muestreo por conveniencia, donde las empresas (proveedores automotrices) se eligieron de acuerdo a su fácil disponibilidad (Mejía, 2014). Se buscó en todo momento que las organizaciones tuvieran una relación comercial con la armadora General Motors (GM). El análisis y procesamiento de la información se realizó a través de una transcripción manual (desgravada de entrevistas) y ordenada en esquemas en función de las preguntas que guiaron la entrevista.

La entrevista consta de dos apartados: 1) información general de la organización y 2) de la relación con empresas del sector automotriz y su vinculación con otras

\footnotetext{
La norma ISO/TS 16949 es el desarrollo de un sistema de gestión de calidad con el objetivo de una mejora continua enfatizando en la prevención de error y en la reducción de desechos de la fase de producción. TS 16949 se aplica en las fases de diseño/desarrollo de un nuevo producto, producción y cuando sea relevante, instalación y servicio de productos relacionados con el mundo de la automoción. Está basado en el estándar ISO 9000. Los requisitos son aplicables a lo largo de toda la cadena de producción. En la actualidad también las plantas armadoras de vehículos se están confrontando con esta norma del ISO.
} 
instituciones sociales. Las preguntas versaban sobre el conocimiento de por qué deciden instalarse en la región potosina, su origen de capital, tamaño de la empresa y sus productos que manufactura, así como también, información sobre sus clientes, proveedores, su vinculación con los organismos gubernamentales académicos, y asociaciones empresariales, también para que expresaran su punto de vista con respecto a sus expectativas en el mercado del sector automotriz, y recomendaciones para generar un encadenamiento productivo en el Estado.

\subsection{Encadenamiento productivo de la industria automotriz en SLP}

Conocer los encadenamientos existentes, sus miembros, sus interrelaciones y sus fuentes de ventaja competitiva es un elemento importante para comprender el desarrollo de las economías locales.

\subsubsection{Organismo gubernamental}

Para que se dé un encadenamiento productivo en la industria automotriz del Estado de SLP es necesario que la formulación de sus políticas industriales las realice el gobierno y los sectores productivos locales, ya que de esta manera, se podrán cubrir realmente las necesidades para lograr el desarrollo de los actores económicos del Estado.

En el caso del gobierno estatal potosino, las acciones que se llevan a cabo comienzan en la SEDECO, organismo que se encarga de promocionar al Estado en los ámbitos local, nacional e internacional, para atraer inversiones a las áreas económicas donde se percibe mayor crecimiento y pueden ser motores de desarrollo, como automotriz, aeroespacial, metalmecánica, eléctrico-electrónico, textil, entre otras. Para lograr esas inversiones es necesario el apoyo de los tres niveles de gobierno y de los sectores productivos del Estado, para crear una base sólida local en cuanto a mano de obra, infraestructura, proveedores, etcétera.

Las acciones se realizan a través de la Dirección General de Promoción Industrial, unidad de la SEDECO que se encarga de ofrecer incentivos fiscales y no fiscales a los inversionistas que se van a instalar en el Estado. Otra de las funciones de dicha Dirección es conseguir recursos federales para que se canalicen a los programas productivos.

Esta unidad gubernamental está dirigida apoyar o incentivar la instalación de la gran empresa que detone el desarrollo del sector industrial y, por consiguiente, de la economía local. En la entrevista realizada al encargado de la Dirección, él señala que 
también han llegado a la Secretaría, microempresarios para solicitar apoyo y han sido canalizados a la Dirección General de Apoyo a la Micro, Pequeña y Mediana Empresa para que reciban todo tipo de información y se realicen las gestiones necesarias para que puedan acceder a los programas de apoyo que ofrece la Secretaría.

La SEDECO manifestó que los proveedores que están siendo empleados por las compañías multinacionales y armadoras; éstas son de manufactura y de servicios, por lo que la Dirección General de Promoción Industrial busca apoyar primero a los proveedores de manufactura y de servicios industriales, después promoverá el desarrollo de la Mipyme local dedicada al material productivo, para que suba niveles en la cadena de proveedores del sector automotriz.

Para lograrlo, la SEDECO, por medio de la mencionada Dirección, y a través de programas federales, Estatales y privados, está llevando acciones en el sector automotriz, por señalar una de las más primordiales, es la de consolidar el clúster automotriz. Durante 2014 se firmaron convenios con empresarios proveedores y terminales del sector automotriz, asociaciones empresariales y centros educativos. Esto con la finalidad de apoyar, promover y desarrollar la competitividad en la región y su despegue en lo económico y social.

La dirección, maneja los proyectos locales del Estado, los promociona para atraer inversiones y ayuda con la gestión a las empresas establecidas o en vías de hacerlo en la región. En estas acciones se ha apoyado sobre todo en la infraestructura, por ejemplo, en el servicio de agua, gas, electricidad, permisos estatales y municipales; a la vez como gestores de manera oficial de los proveedores del sector automotriz.

Por último, esta División se encarga de informar a los integrantes del clúster automotriz sobre los programas que se ofrecen en el Estado para apoyarlos en su desarrollo, crecimiento y producción, para que sean más competitivas en la cadena de proveeduría de la industria automotriz.

Para que se alcancen los objetivos propuestos por las políticas de fomento industrial existe un elemento importante: la participación del empresario local. En la entrevista se abordó el tema de la cultura empresarial, para conocer cómo estos organismos perciben al empresario potosino actualmente.

La idea general que se tuvo, de acuerdo con las respuestas, fue que a pesar de que se comienza a percibir una actitud de cambio en el empresario local, ésta no ha sido suficiente y, sobre todo, ha llegado demasiado tarde. 
El paternalismo y la mentalidad de obtener ganancias en el corto plazo, la falta una cultura de riesgo y de más trabajo son algunas de las causas por las que el empresario no ha podido desarrollarse, sobre todo en el sector industrial. Los organismos también esperan que los empresarios tengan una actitud más proactiva, que de ellos salgan las iniciativas.

Por último, en cuanto a la calidad, señalaron que los empresarios no están acostumbrados a tener los niveles de competitividad y de calidad que está requiriendo las grandes empresas y por supuesto la industria automotriz, algo muy importante, les falta mucha vocación de servicio.

\subsubsection{Empresas automotrices}

De acuerdo con la entrevista a empresarios y/o funcionarios de empresas proveedoras (multinacionales) del sector, se puede confirmar que son las estrategias comerciales de cada empresa las que determinan su localización en algún punto del planeta, el caso de SLP no es la excepción, ya que es una plaza que puede representar reducción de costos, por ejemplo, en la localización geográfica, pues se encuentra en un punto que puede tener acceso rápido a otros países por su cercanía a la frontera con Estados Unidos y un puerto por lo menos. La localización de las empresas de alta tecnología en la región potosina no obedece a algún programa específico del gobierno, sino a sus propias estrategias de expansión.

Con respecto a la ubicación de sus clientes, se observa que en el caso de algunos proveedores su producción no depende exclusivamente de la demanda de GM., mencionaron tener al menos un cliente en el país o en el extranjero. En tanto que para los proveedores de segundo y tercer nivel es evidente que sus capacidades no les han permitido abarcar más mercado que el local.

En el caso de los proveedores de niveles más bajo (tier 3 y 4), no existe vínculo con terminales, lo que quiere decir que no hay encadenamiento productivo; sin embargo, han establecido vínculos con los proveedores de nivel tier 1 y 2 . Sobre por qué no se han vinculado con los proveedores de primer nivel o la armadora, algunos contestaron haber tenido obstáculos para conformar encadenamientos productivos con esas empresas, debido principalmente a la falta de interés por parte de la empresa multinacional o la empresa terminal (armadoras) de tener una relación comercial y de exigencias de calidad y costo en los productos y/o servicios. Para establecer relaciones con proveedores de primer nivel, y plantas terminales, entre otros organismos, recurren a las asociaciones o cámaras empresariales, lo que muestra que 
por ahí se podría comenzar a dar el enlace entre el proveedor productivo local y la gran empresa. Sólo han recurrido al gobierno para solicitar e informarse sobre apoyos financieros. Al respecto los empresarios entrevistados de organizaciones de Tier 2 y Tier 3, en su totalidad, manifestaron que no han recibido o reciben ningún tipo de apoyo del gobierno.

Acerca del apoyo que brindan las instituciones de gobierno, académicas, y organismos privados, los proveedores actuales de segundo y tercer nivel (tier 2 y 3 ) coinciden en que el ofrecido por las instituciones educativas es bueno, sobre todo para reclutar y capacitar a su mano de obra. Para estos proveedores, el apoyo que brindan las asociaciones empresariales es mejor que el del gobierno en sus tres niveles, esto debido a que muchas veces han recurrido a los organismos gubernamentales solicitando información de los programas y han tenido muchos problemas para que los tomen en cuenta o le den seguimiento a sus casos, lo que ha generado en ellos cierta desconfianza en cuanto a la eficacia y veracidad de los apoyos que ofrecen.

En cuanto a las acciones llevadas a cabo por los organismos gubernamentales y privados que han influido en sus relaciones con proveedores o clientes, señalaron que los eventos organizados por las asociaciones empresariales, seminarios, reuniones y ferias que organiza el gobierno, son los que mejor les han funcionado, debido a que en este tipo de eventos es donde logran tener contacto directo entre ellos.

Por último, para conocer la difusión de los programas de fomento industrial que ofrece el gobierno en los tres niveles (municipal, estatal y federal), así como qué tanto se está aprovechando, los entrevistados de empresas Tier 2 y Tier 3, señalaron que no han recibido apoyo en lo tecnológico, innovación, económico, calidad y/o fomento a la integración de empresas. En las empresas Tier 1, el 100\% de los funcionarios entrevistados, señalaron que en algún momento si han recibido apoyos de diferente índole por parte del gobierno. En el caso de las empresas de niveles Tier 2 y 3 manifiestan que no han recibido apoyo alguno o no tenían conocimiento de ellos, al respecto uno de los proveedores potenciales señaló textualmente lo siguiente:

"Mira, te voy a decir una cosa, yo creo que con muy poco temor a equivocarme, que todas las personas que ustedes han entrevistado en esta situación, deben andar en el mismo rango de conocimiento de programas de gobiernos del estado ofederales o municipales, entonces quiero decir con esto, que casi todo mundo los desconocemos y/o no hemos recibido ningún tipo de apoyo por parte del gobierno" 
En lo que se refiere a sus expectativas, se les preguntó acerca de lo que esperan de los proveedores, clientes, gobierno y de los egresados de las instituciones educativas. Para el caso de los proveedores de todos los niveles, comentaron que debido al arribo de muchas empresas foráneas, aumentará la competencia en el Estado, lo que puede traducirse en que se estandaricen servicios y sistemas productivos además surjan nuevas opciones, se conformen alianzas entre proveedores locales para ofrecer mejor servicio, mayor calidad y buen precio; pero en lo que hicieron énfasis fue en que el empresario local debe cambiar todavía más su actitud, debe tener capacidad de compromiso, más arrojo y mejorar su calidad en el producto y servicio.

Del gobierno esperan que los siga apoyando y que incentive el desarrollo de la tecnología local. También que esta vez haya mayor difusión de los programas y servicios que ofrecen, y que las acciones realizadas sean de largo plazo y tengan un efecto real en el desarrollo del empresario local, sobre todo en este caso del sector automotriz y para los proveedores de niveles dos y tres.

Respecto a la mano de obra, señalaron que aunque saben que hay mano de obra capacitada y especializada, todavía no es suficiente, lo que en el mediano plazo se puede traducir en un déficit de mano de obra, sobre todo en competitividad del sector automotriz. La mayoría de los entrevistados de empresas Tier 1 y 2, señalaron que falta mayor vinculación con las universidades, ya que los alumnos son los que tienen que venir a las empresas a solicitar sus prácticas profesionales y no la institución educativa para que se concrete a través de convenios o programas de vinculación. Todos los entrevistados enfatizaron en que hace falta programas educativos enfocados a sistemas productivos del sector automotriz. También manifestaron los entrevistados, que la Universidad se acerque más a los empresarios para que tengan conocimiento de las necesidades tecnológicas y de personal, y sobre ello trabajar en sus programas educativos.

\section{Instituciones educativas}

Las universidades y escuelas tecnológicas forman parte de las instituciones que están tratando de adaptar sus planes y proyectos educativos a la demanda potencial del clúster mediante la creación de convenios y programas educativos, para formar estudiantes competitivos y especialistas en alguna área específica.

La Universidad Politécnica de San Luis Potosí [UPSLP], es una institución de educación superior pública creada en 2001, dotada con personalidad jurídica, patrimonio público y tiene por objetivo: impulsar e impartir la educación superior; organizar, fomentar, realizar investigación y desarrollo tecnológico, científico, 
humanístico, difundiendo el conocimiento y la cultura, que contribuyan a impulsar, diversificar y equilibrar el desarrollo regional, estatal y del país.

En la entrevista realizada a dos funcionarios de esta casa de estudios se puede señalar lo siguiente: Entre su oferta educativa relacionada con el sector automotriz, tienen las carreras de: Ingeniería en Sistemas y Tecnologías Industriales, Ingeniería en Telemática e Ingeniería en Tecnologías de Manufactura. La UPSLP, cuenta con una Dirección de Servicios Escolares y Vinculación. En la actualidad la Universidad tiene convenios de vinculación y desarrolla proyectos de investigación en los que colaboran profesores y estudiantes, con la finalidad de impulsar el desarrollo de competencias profesionales de los estudiantes en sus diferentes carreras.

El proceso de vinculación con empresas del sector automotriz es a través de las prácticas profesionales de los estudiantes y que estas las realizan en los últimos semestres de su carrera, de sexto semestre en adelante. Los alumnos, para poder realizar sus prácticas profesionales, sus estudios y asignaturas las cursan por la tarde, para que por las horas de la mañana puedan incorporarse a una empresa con las que se tienen convenio. La universidad carece de laboratorios en áreas de especialización del sector automotriz, por lo tanto, que mejor laboratorio que las propias empresas. Para poder ingresar algunas de las empresas o planta de manufactura del sector automotriz, se requiere que los estudiantes tengan un promedio de 8.5 en adelante y manejen en un $70 \%$ el idioma inglés. Se considera que al estar realizando prácticas profesionales en estas empresas se genera un compromiso (como cualquier empleado de la empresa), y de la cultura organizacional. Los convenios en los que participa la UPSLP con empresas para las prácticas profesionales de los alumnos, es porque un gran porcentaje $60 \%$ de los egresados de esta institución se encuentran laborando y en puestos directivos en estas empresas. El $90 \%$ de los estudiantes en el noveno semestre de su carrera, se encuentra laborando bajo un contrato legal.

La UPSLP ha realizado varios convenios, con los diferentes sectores de la sociedad, tanto regional, como nacional e internacional. La Institución tiene convenios con varias empresas del sector automotriz, vinculación que se lleva a cabo a través de programas de capacitación, formación profesional e investigación. Por mencionar algunos de las relaciones que se tienen con este sector automotriz, son las siguientes:

Con la empresa Asea Brown Borevi $[A B B]$, líder mundial en tecnologías de energía y automatización, se firmó un convenio para llevar a cabo proyectos tecnológicos con sentido social. ABB otorgará tres becas por año, hasta llegar a un máximo de ocho becas simultáneas para estudiantes sobresalientes de Ingeniería. Las becas que otorga esta Cía., van más allá de un apoyo económico a los estudiantes, pues tienen la 
oportunidad de participar en eventos regionales o internacionales de la empresa, así como acceder a residencias en diversas plantas o localidades de ABB en todo el mundo durante períodos vacacionales y la posibilidad real de obtener empleo dentro de la compañía al final de su carrera.

En el área de proyectos académicos, la empresa Freescale colabora en conjunto con la UPSLP para desarrollar futuras aplicaciones tecnológicas en el área de semiconductores. Esta colaboración proporciona a los estudiantes de Telemática una enriquecedora experiencia, con el conocimiento y habilidades para trabajar en la industria de semiconductores y una mejor inserción en el mercado laboral.

Desde el punto de vista institucional, señalan los entrevistados, que la vinculación contribuye en los estudiantes a la adquisición de experiencia y desarrollo de habilidades necesarias en esta área del conocimiento, por ello, se tiene relación con empresas de primer y segundo nivel, que permita el desarrollo y aplicación de la teoría y la práctica, y la oportunidad de poder desarrollar un trabajo recepcional acorde a la realidad laboral. Con las empresas del sector automotriz que se tiene vinculación son: PGI; Leider Ryder, Draexlmaier, Valeo, Cummins Motors, Comtitec Mexicana, Continental y Remy, entre otras.

Por otra parte, el Colegio de Educación Profesional Técnica del Estado de San Luis Potosí [CONALEP] es una institución educativa de Nivel Medio Superior que forma parte del Sistema Nacional de Educación Tecnológica, con personalidad jurídica y patrimonio propio, con el objetivo de contribuir al desarrollo estatal mediante la formación de recursos humanos calificados y de la superación profesional del individuo. El funcionario que se entrevistó, señala que el Colegio trabaja para que los estudiantes se realicen como profesionales técnicos capaces de laborar en cualquier empresa, con base en un modelo educativo basado en competencias laborales certificadas. La institución ofrece formación Profesional Técnico-Bachiller a través de su oferta educativa de doce diferentes carreras técnicas. Las carreras que se relacionan con el sector automotriz son las siguientes: Control de Calidad, Electricidad Industrial; Electromecánica industrial, Metalmecánica y Productividad industrial.

La relación que se tiene con empresas de manufactura es a través de las prácticas profesionales de los alumnos. El estudiante a partir del $5^{\circ}$ semestre puede comenzar con sus prácticas profesionales ( $370 \mathrm{hrs}$.), estas se formalizan mediante un convenio de colaboración que firma el Director General (Delegado de CONALEP en el Estado) y el Gerente General y/o Administrativo de la empresa, con este convenio se aceptan responsabilidades y derechos de ambas partes. En el $6^{\circ}$ semestre los alumnos realizan su servicio social, dado que la institución es un organismo público descentralizado 
del Gobierno, este servicio social en su mayoría de los casos, los alumnos lo realizan en instituciones del sector público.

En el convenio de colaboración entre las partes (CONALEP-empresa) se estipula algunas consideraciones para la formación de los estudiantes, por señalar algunas: acceso a los estudiantes a las instalaciones de la planta, con el objetivo de obtener un aprendizaje en la observación, explicación de los mismos trabajadores y el sentido de pertenencia a la cultura organizacional. También se estipula en el convenio, que los estudiantes reciban algún tipo de apoyo económico por parte de las empresas donde realizan sus prácticas profesionales, que en la mayoría de estas empresas (99\%), si se les otorga un estímulo económico.

El CONALEP desarrolla un proyecto denominado "Trayectos Técnicos", este proyecto es un apartado de módulos o materias que tienen acceso los alumnos y que ayudan a la formación profesional en áreas o tareas muy específicas, por ejemplo, con GM donde esta armadora maneja maquinaria que no se conocía en el Estado, a través de los "Trayectos Técnicos" se les enseña a los estudiantes a manipularla, con apoyo de los técnicos de la armadora GM, y así los estudiantes se especializan en manejar una determinada máquina y obtener una formación que requiere el sector productivo automotriz. En un estudio de factibilidad realizado por la Universidad Politécnica de San Luis Potosí en el 2013, señala en sus resultados, que un 70\% de los egresados de CONALEP-SLP., constituye la base operativa de la industria automotriz potosina.

Por otro lado, la vinculación también se realiza por medio de educación continua, con cursos de capacitación para empresarios, empleados y obreros. Estos cursos o talleres, son solicitados por pequeñas y medianas empresas locales mexicanas. Se capacita a gerentes de mandos medios y alta gerencia. Cursos para obreros y operarios del sector industrial, específicamente en el automotriz. Se cuenta con un Departamento de capacitación en los diferentes planteles del Estado. Entre las principales empresas del sector automotriz con que se tiene convenio de colaboración, son las siguientes:

- Cummins. Empresa que fabrica cigüeñales para motores ISB y cabezas para motores.

- EKK Eagle Industry México. Empresa que fabrica válvulas y sellos mecánicos para la industria automotriz.

- Metalsa. Desarrolla una gran cantidad de productos estructurales para autos ligeros y pesados.

- General Motors. Armadora establecida en San Luis Potosí. 
Como resultado de la información proporcionada por los diferentes directivos de las instituciones educativas investigadas, se puede concluir lo siguiente: Con respecto a la relación que se tiene con el gobierno, federal, estatal y municipal, para proyectos de articulación productiva en el sector automotriz, los entrevistados de las dos instituciones educativas, señalan que es nula la vinculación con organismos del gobierno de cualquier nivel. La UPSLP señaló que solamente en una ocasión el gobierno estatal los invitó a impartir un curso de capacitación a empleados de nivel medio de la armadora GM-SLP. Ambas instituciones manifestaron que desconocen la conformación y/o consolidación del clúster automotriz en SLP, o que a estos organismos no se les invito a participar.

Se deduce que la vinculación con el sector empresarial constituye un ámbito fundamental en las escuelas, el cual les aporta las herramientas necesarias para atender los requerimientos provenientes de todos los sectores de la sociedad mediante una formación profesional pertinente, acorde con dichas necesidades. Además, establece los puentes necesarios de comunicación e intercambio entre sectores, que facilita a los alumnos del nivel superior y técnico su inserción en los sectores social y productivo; así poder contribuir a su dinamismo y fortalecimiento de manera activa y comprometida.

Con base en las entrevistas realizadas, se concretaron las áreas estratégicas de actuación, las necesidades y las acciones a emprender en la articulación productiva del sector automotriz del Estado potosino (tabla 3):

\section{Conclusiones}

La participación del sector automotriz en el desarrollo económico de México ha sido constante desde principios del siglo XX y sigue marcando la pauta en la segunda década del siglo XXI, la posición estratégica de nuestro país en el contexto internacional gracias a los recursos naturales con los que cuenta, a la capacidad y bondad de la mano de obra y a condiciones de logística y operaciones en algunas zonas de la república favorece la fabricación de vehículos ligeros y pesados por lo que la ubicación de las plantas ha detonado la derrama económica para la mejora de las condiciones de la población.

Una de las conclusiones, es que las empresas automotrices de SLP se desarrollan hoy en un contexto de globalización, donde las actividades económicas no sólo se internacionalizan sino que además están integradas funcionalmente debido a una coordinada división global del trabajo. 
El complejo automotriz de SLP es encabezado por capitales de diferentes países y han desarrollado especialidades productivas distintas, esto se debe en buena medida a los antecedentes industriales de esta ciudad, a su localización geográfica y particularmente al tipo de plantas automotrices y de otros equipos de transporte que llegaron en 2002 al Estado. Con la llegada de GM, en 2008, SLP empezó a convertirse en sitio atractivo para producir partes para el motor y la transmisión y partes de estampado, de la carrocería y del chasis, se convirtió en la localización óptima de las maquilas productoras de partes eléctricas y electrónicas, en particular las que producen arneses y sus componentes. Esto definió también el origen de la inversión que hoy predomina en la región; son las estadounidenses las que concentran la mayoría, aunque recientemente las orientales son las que han invertido en mayor medida.

Si recuperamos la definición inicial de clúster, como una concentración sectorial y geográfica de empresas con relaciones de subcontratación entre ellas, que integra los apoyos institucionales a las empresas y las economías internas y externas (Humphrey y Schmitz, 1995; Porter, 1991), podemos decir que en la Entidad potosina encontramos un clúster automotriz en formación. Como se ha constatado, en la experiencia tanto la cadena productiva como las instituciones de apoyo (gobierno, academia) del clúster requieren ser fortalecidas.

Otra conclusión, es que los encadenamientos hacia atrás no los están generando o participan muy poco en ellos las empresas Tier 1 que mantienen una relación más cercana y exclusiva con la ensambladora y las que son exportadoras directas, es decir, las proveedoras exclusivas de GM-SLP. Los productores que están desarrollando la cadena hacia atrás, son los proveedores no exclusivos y los que no le venden a esta armadora, son el segmento de proveedores (Tier 1 y Tier 2 y Tier 3), de empresas pequeñas o medianas. Es, en estos proveedores donde debe enfocarse la atención y dirigirse las políticas estatales y locales de fomento de encadenamientos productivos. Es importante considerar que se propone diseñar políticas que no partan de una concepción que tome como situaciones excluyentes fomentar empresas exportadoras o solamente dedicadas al mercado interno y/o externo. En el caso estudiado encontramos empresas proveedoras de niveles inferiores que han desarrollado capacidades para participar y competir tanto en el mercado externo como en el interno para mantener como clientes a diferentes empresas y corporativos, siempre y cuando no mantengan una relación de exclusividad y mantengan relativa independencia de las ensambladoras o terminales.

Como vemos el programa de fomento económico basado en clústeres apenas inicia en SLP con muy poco tiempo como para poder evaluar sus resultados, sobre todo en lo relacionado con la creación y apoyo a empresas locales o regionales que se integren 
a la cadena de proveedores. Sin embargo, cuenta con algunos elementos locales que actúan en su favor. Por ejemplo, cuenta con un Centro de Tecnología Avanzada [CIATEQ] creado en 2002 que proporciona servicios tecnológicos a las empresas, existen al menos dos empresas de maquinados y otra que produce diferentes tipos de soportes metálicos. Una de estas empresas, que es pequeña, se creó en el año 1987, para ello no recibió apoyos de la Secretaría de Economía [SE], forma parte de la cadena productiva y está aprovechando la vinculación con instituciones educativas de nivel superior para tener personal capacitado a bajo costo a través de becarios. Experiencias como ésta pueden multiplicarse.

Otra de las conclusiones obtenidas en esta investigación es que no se ha logrado generar un encadenamiento productivo alrededor de la industria automotriz, debido a que las políticas y acciones de los organismos gubernamentales no han sido suficientes para apoyar el desarrollo de las capacidades productivas del empresario.

En lo que se refiere a las políticas de fomento industrial, durante muchos años estuvieron enfocadas a crear las condiciones en el estado para la instalación de la gran empresa nacional o extranjera dedicada a la exportación, dejando de lado las necesidades de los empresarios locales o proveedores potenciales para que pudieran vincularse con la gran empresa en sus procesos productivos.

Al respecto, es importante señalar que los Planes de Desarrollo y Programas Industriales, en los ámbitos federal y estatal, no llegaron a los empresarios locales, lo cual se reflejó en la baja participación de insumos de fabricación nacional en los productos finales que se dirigían a la exportación. En cuanto a las acciones, y con base en los resultados obtenidos de las entrevistas, se pudo confirmar que el sector empresarial comienza a participar junto con el gobierno en la formulación de políticas industriales, aunque todavía hace falta el apoyo al desarrollo de las Mipymes.

Otro hallazgo en la investigación es que la cultura empresarial por muchos años en el Estado de SLP, ha sido la de invertir en sectores económicos de bajo riesgo y en los que puedan obtener ganancias en el corto plazo, como es el de servicios. Es únicamente en este sector donde el microempresario local ha podido formar parte del grupo de proveedores de las empresas del sector automotriz del estado.

Actualmente se intenta, con gran avance, conformar encadenamientos productivos. Los actores económicos locales, tanto públicos como privados, se están organizando para diseñar una nueva política industrial que apoye e impulse al empresario local para insertarse en las cadenas productivas de los nuevos proyectos del estado y, en especial, el automotriz en la ciudad potosina. 
En cuanto al involucramiento de las instituciones educativas en la cadena productiva, el gobierno del Estado es el responsable de crear políticas de fomento a la vinculación entre la academia con los sectores productivos y sociales para elevar la calidad de la educación, en consecuencia, una forma para contribuir a la formación y generación de mano de obra calificada, especializada y por supuesto de empleo de calidad.

A partir de los resultados obtenidos de la investigación, se recomienda implementar amplias campañas para difundir los programas y servicios que ofrecen los organismos gubernamentales y privados que están interviniendo en la promoción y desarrollo industrial, pues sólo de esta manera podrán llegar hasta los proveedores de niveles inferiores, participando en la cadena productiva de un sector industrial. Así mismo, se recomienda que el apoyo que se empieza a brindar a los pequeños empresarios potosinos sea real y efectivo, se dé seguimiento a los casos particulares hasta que logren conectarse en la cadena de proveedores de materia prima de la industria automotriz del estado.

Por último, el impulso y apoyo a las cadenas productivas debe ser real y evitar la simulación, además es necesario que el apoyo sea transparente y se canalice aquellos empresarios locales que lo necesitan para elevar sus niveles de competitividad y puedan participar en el proceso productivo de la industria automotriz.

Tabla 1. Ventas al Mayoreo de Automóviles en México y en el Estado de San Luis Potosí 2000-2010 (Número de unidades).

\begin{tabular}{|c|c|c|}
\hline ÃNO & MÉXICO & SAN LUIS POTOSI \\
\hline 2000 & 603,027 & 9,146 \\
2001 & 674,033 & 10,157 \\
2002 & 722,257 & 12,440 \\
2003 & 692,627 & 12,345 \\
2004 & 739,862 & 13,428 \\
2005 & 701,208 & 12,521 \\
2006 & 688,653 & 12,158 \\
2007 & 620,256 & 10,492 \\
2008 & 583,063 & 10,828 \\
2009 & 424,278 & 9,574 \\
2010 & 515,055 & 10,027 \\
\hline
\end{tabular}

Fuente: elaboración propia con base a datos de la Industria Automotriz en México, Instituto Nacional de Estadística, Geografía e Información INEGI 2012. 
Tabla 2. General Motors de México, S. de R. L. de C.V. (Complejo San Luis Potosí)

\begin{tabular}{|c|c|}
\hline Tipo de Industria & Terminal (Ensambladora) \\
\hline Superficie total de S.L.P. & $\begin{array}{l}347 \text { hectáreas de superficie }=855 \text { acres } 219,000 \mathrm{~m} 2 \\
\text { construcción de la planta de } 14 \text { meses. }\end{array}$ \\
\hline Inicio de Operaciones: & junio de 2008 \\
\hline Empleados: & 2,311 \\
\hline \multirow[t]{2}{*}{ Origen de la Inversión: } & Estados Unidos de América \\
\hline & $\begin{array}{l}\text { Arranque de Producción: } \\
\text { - Planta Ensamble (2008) } \\
\text { - } \text { Planta de Estampado (2008) } \\
\text { - Planta de Transmisiones (2009) } \\
\text { - } \quad \text { Expansión de Planta Ensamble SUV (2012) }\end{array}$ \\
\hline Plantas: & $\begin{array}{l}\text { Arranque de Construcción: } \\
\text {-Planta Transmisiones de Doble Embrague }\end{array}$ \\
\hline Productos: & $\begin{array}{l}\text { Vehículos: } \\
\text {-Chevrolet Aveo (2008) } \\
\text {-Chevrolet Trax (2012) }\end{array}$ \\
\hline
\end{tabular}

Fuente: elaboración propia con base en la investigación de campo a la armadora, 2014.

Tabla 3. Vinculación entre gobierno, empresas y universidades

\begin{tabular}{|c|c|}
\hline Necesidades detectadas & Acciones recomendadas \\
\hline $\begin{array}{l}\text { - Una institución permanente del sector en el } \\
\text { Estado, con la participación de la academia, } \\
\text { el gobierno, y las empresas, para poder } \\
\text { desarrollar y dedicar recursos a planes } \\
\text { específicos. } \\
\text { - Mayor colaboración y vinculación de las } \\
\text { instituciones educativas con el sector } \\
\text { gobierno. }\end{array}$ & $\begin{array}{l}\text { - Consolidar el Clusters automotriz. } \\
\text { - Promover, diseñar y llevar a cabo programas } \\
\text { y proyectos educativos entre gobierno y } \\
\text { escuela relacionados con el sector } \\
\text { automotriz y otros sectores productivos. }\end{array}$ \\
\hline \multicolumn{2}{|l|}{ Desarrollo de personal calificado y especializado } \\
\hline Necesidades detectadas & Acciones recomendadas \\
\hline $\begin{array}{l}\text { - Personal técnico bien capacitado. } \\
\text { - Especialistas dedicados a la problemática de } \\
\text { la industria automotriz. } \\
\text { - Vinculación escuela-empresa. }\end{array}$ & $\begin{array}{l}\text { Identificar los perfiles necesarios de personal } \\
\text { especializado por parte de las empresas. }\end{array}$ \\
\hline
\end{tabular}


Tabla 3. Vinculación entre gobierno, empresas y universidades

\begin{tabular}{|c|c|}
\hline \multicolumn{2}{|c|}{ Desarrollo y fomento de la investigación y desarrollo } \\
\hline Necesidades detectadas & Acciones recomendadas \\
\hline $\begin{array}{l}\text { - Centros de I\&D enfocados al desarrollo de } \\
\text { la industria automotriz. } \\
\text { - Más convenios entre empresa y tecnología } \\
\text { propietaria por empresas mexicanas. } \\
\text { - Centro de desarrollo tecnológico para la } \\
\text { industria vinculado con las instituciones de } \\
\text { Educación Superior. }\end{array}$ & $\begin{array}{l}\text { - Seguir estrategias de otras regiones que han } \\
\text { desarrollado gestiones en la cooperación y } \\
\text { desarrollo de la investigación. } \\
\text { - Comunicar al gobierno del Estado la } \\
\text { necesidad de elaborar y revisar, a nivel } \\
\text { federal, un plan de incentivo para desarrollar } \\
\text { la investigación. }\end{array}$ \\
\hline \multicolumn{2}{|c|}{ Desarrollo competitivo de proveedores de autopartes (Tier $1,2,3)$} \\
\hline Necesidades detectadas & Acciones recomendadas \\
\hline $\begin{array}{l}\text { - Formación en calidad y productividad en } \\
\text { proveedores de segundo y tercer nivel. } \\
\text { - Apoyo gubernamental para el desarrollo de } \\
\text { proveedores de segundo y tercer nivel. } \\
\text { - Proveedores confiables en lo referente a } \\
\text { calidad y entrega. } \\
\text { - Apoyo a empresas Mexicanas para su } \\
\text { certificación en el recicladoy transformación } \\
\text { de desechos industriales. }\end{array}$ & $\begin{array}{l}\text { - Generar el clúster automotriz. } \\
\text { - Establecer plan de formación en calidad } \\
\text { automotriz para proveedores. } \\
\text { - Más vinculación y relación entre plantas } \\
\text { terminales y proveedores de segundo y tercer } \\
\text { nivel. } \\
\text { - Políticas Públicas enfocadas al control del } \\
\text { medio ambiente. }\end{array}$ \\
\hline
\end{tabular}

Fuente: elaboración propia con base en los resultados de la investigación de campo (empresas, gobierno e instituciones educativas) en el Estado de SLP 2014.

\section{Referencias Bibliográficas}

Dávila, A. (2002). Impactos económicos del TLCAN en la frontera Norte de México. En Rafael Fernández de Castro y Beatriz Leycegui (coords), TLCAN ¿Socios naturales? Cinco años del Tratado de Libre Comercio de América del Norte, México, ITAM-Miguel Ángel Porrúa, 177-224.

Feser, E., y Bergman, E. (2000). National industry cluster templates: a framework for applied regional cluster analysis. Regional studies, 34(1), 1-19. DOI: 10.1080/ 00343400050005844

Flores, B. (2013). La integración de un clúster ¿un alternativa para la industria artesanal del Ónix y Mármol? (Tesis de Maestría). Recuperada de http:// www.biblio.colpos.mx:8080/jspui/handle/10521/2084 
Gil, F. (2011). Algunos Interrogantes en torno al desarrollo de los clústeres. Revista Ciencias Estratégicas, 19(26), 281-293.

Hernández, R., Fernández, C. y Baptista, P. (2010). Metodología de la investigación ( $7^{\mathrm{a}}$ ed.). México: Editorial Mc Graw-Hill.

Hualde, A. y Gomis, R. (2007). PYME de Software en la Frontera de México: Desarrollo Empresarial y Construcción Institucional de un Clúster. Revista Latinoamericana de Economía. Problemas del Desarrollo. 38(150), 193-272.

Instituto Nacional de Estadística, Geografía e Información (INEGI) (2012). La industria automotriz en México 2012.

Lara Hernández, R. (2010). Conformación del clúster textil en Michoacán desde el enfoque del desarrollo local. (Tesis Doctoral). Universidad Michoacana de San Nicolás de Hidalgo, México.

Moreno, A., Ríos, D., Alva, B., y Medina, R. (2008). El sistema de innovación regional de San Luis Potosí. Avances y retos. SinncO 2008. Recuperado de http:// www.concyteg.gob.mx/formulario/MT/MT2008/MT2/SESION3/ MT2_MORENO_RIOS_ALVA_MEDINA.pdf

Mejía Navarrete, J. (2014). Perspectiva epistemológica de la investigación social en América Latina. Estudios de Sociología, 1(14), 37-60.

Porter, M. (2003). Ser competitivo: nuevas aportaciones y conclusiones. Bilbao: Ediciones Deusto, 1-473.

SEDECO. Secretaría de Desarrollo Económico del Estado de San Luis Potosí. (2014). Industria Automotriz y de autopartes del Estado de San Luis Potosí-2012/2014. Gobierno del Estado de San Luis Potosí.

Secretaría de Hacienda y Crédito Público (SHCP-NAFIN). (2011). Informe sobre la evolución y resultados de la Banca de desarrollo, primer semestre 2011.

Sölvell, Ö. (2009). Clúster equilibrando fuerzas evolutivas y constructivas. Suecia: Danagårds Grafiska, Ödeshög, 1-116.

Vera Garnica, R. y Ganga Contreras, F. A. (2007). Los clústers industriales: precisión conceptual y desarrollo teórico. Cuadernos de Administración, 20(33), 303-322. 
